\title{
Aprendiendo de forma cooperativa a través del método científico
}

María Dolores Mauricio $^{a}$, Eva Serna ${ }^{b}$, Soraya L. Vallés ${ }^{a}$, Martín Aldasoro ${ }^{a}$ y José M. Vila ${ }^{a}$

${ }^{a}$ Departamento de Fisiología, Universitat de Valencia. M.dolores.mauricio@uv.es, Lilian.valles@uv.es, aldasoro@uv.es y vila@uv.es. ${ }^{b}$ Departamento de Patología, Universitat de Valencia. Eva.serna@uv.es

\begin{abstract}
Usually, students tend to divide tasks unequally when they have to work in groups, everyone prepares a part of the manuscript and later they bind all parts together and deliver it. In this way, learning is fragmented and the desired goal is not achieved: to work cooperatively. In our experience we proposed students to work as a group of scientists. From a brief background they had to write an introduction, design a protocol using the indicated experimental technique and finally they had to speculate and discuss on the expected results. The activity should have raised the cooperation among all group members, because the correct understanding of a section required of the understanding of the preceding sections. The results showed that our students have problems working as a team, because only students with high motivation for the subject showed an improvement in learning by this method. This is probably due to an education system that qualifies students based on their individual effort. However, the activity encouraged many positive aspects such as doubts clarification, discussion of ideas among them, the correct search for information and also offered quality manuscripts.
\end{abstract}

Keywords: cooperative learning, scientific method, skills.

\footnotetext{
Resumen

Habitualmente cuando se plantea una actividad en grupo los alumnos tienden a dividir de forma desigual las tareas, reúnen lo realizado individualmente y lo entregan. De esta manera, el aprendizaje se fragmenta y no se consigue el objetivo deseado: trabajar cooperativamente. Nuestra experiencia consistió en proponer a los estudiantes que trabajasen como un grupo de cientificos. A partir de unos breves antecedentes se les pedia que escribieran una introducción, elaborasen un protocolo experimental usando la técnica indicada y por último que especulasen y discutiesen sobre los resultados esperados según el protocolo diseñado. La actividad planteada debía fomentar la cooperación de todos los integrantes del grupo, pues el correcto entendimiento de un apartado del trabajo requería de la comprensión de los apartados anteriores. Los resultados evidenciaron que el aprendizaje de los conceptos trabajados en grupo mejoró en aquellos alumnos que tenian mayor motivación por la asignatura. En general, trabajar en equipo entraña dificultad para nuestros estudiantes, habituados a
} 
un sistema educativo que califica en base al esfuerzo individual. Sin embargo la actividad fomentó diversos aspectos positivos como la resolución de dudas, el debate de ideas entre iguales, la búsqueda correcta de la información y además ofreció manuscritos de calidad.

Palabras clave: aprendizaje cooperativo, método cientifico, competencias.

\section{Introducción}

Desde la adecuación al Espacio Europeo de Educación Superior (EEES), la actividad docente debe ir encaminada a conseguir que el alumno tenga un papel más activo en el proceso de aprendizaje. Entre las competencias de los estudiantes se encuentra saber trabajar en equipo y tener habilidades de comunicación. El trabajo en grupo implica la cooperación entre los alumnos al compartir un mismo objetivo que tienen que conseguir conjuntamente y ofrece mayores resultados cognitivos que el trabajo individual (Johnson, 1981), también aumenta la motivación intrínseca (Crooks, 1988) (Mauricio, 2015) y la satisfacción general con los esfuerzos del grupo (Rovai, 2002).

Actualmente la práctica docente universitaria se basa en tres pilares: la clase magistral, las clases prácticas y los seminarios. Estos últimos suponen un cambio radical en la metodología docente, pues son los alumnos los ponentes. Se trata de una actividad que complementa las clases teóricas y prácticas y que tiene como finalidad que el alumno participe activamente en la búsqueda de la información, la procese y elabore un trabajo en grupo, mientras el profesor tutela, corrige y dirige. Esta actividad es la que nos permite aplicar una metodología de aprendizaje cooperativo.

Generalmente, cuando diseñamos actividades de trabajo en equipo para nuestros alumnos, estos tienden a dividir de forma desigual las tareas, reúnen lo realizado individualmente y lo entregan. De esta manera, el aprendizaje se fragmenta. La cuestión que nos planteamos es cómo fomentar realmente un aprendizaje cooperativo. Hay diversas estrategias para lograrlo (Johnson, 1999). En primer lugar, los equipos han de estar estructurados (no más de 6 personas y heterogéneos) y con roles bien definidos, orientados a resolver una tarea específica a través de la colaboración. El docente ha de diseñar las actividades de forma que los estudiantes perciban que requieren el esfuerzo coordinado de todos y cada uno de los miembros del equipo para completar la tarea con éxito. Entre las distintitas estrategias que favorecen el aprendizaje cooperativo, encontramos la investigación en grupo. Consiste en un plan de organización en el que los estudiantes divididos en equipos utilizan herramientas como la investigación cooperativa, discusiones grupales o planificación de proyectos. En este sentido, decidimos plantear la actividad de seminarios como la redacción de un proyecto científico. Les propusimos que trabajasen como un grupo de científicos que tenían una hipótesis y disponían de una técnica concreta para resolverla. A partir de unos breves antecedentes y la hipótesis de trabajo, se les pedía que escribieran una introducción, elaborasen un protocolo experimental usando la técnica indicada por los profesores y por último que especulasen sobre los resultados esperados y los discutiesen. De esta manera esperábamos evitar que los alumnos realizasen un mero trabajo monográfico en el que cada miembro escribe una parte sin compartir ni debatir la

(cc) EY-NC-ND 2016, Universitat Politècnica de València 
información encontrada con el resto de compañeros. Con esta modalidad resultaba difícil elaborar cada una de las partes del manuscrito por separado, pues el diseño del protocolo implicaba la interiorización de varios conceptos impartidos en las clases teóricas y que debían figurar en la introducción del trabajo. Asimismo, al redactar los resultados esperados era necesario el entendimiento de la metodología aplicada, que a su vez se fundamentaba en mecanismos fisiológicos estudiados en el temario teórico. El diseño del protocolo experimental era un apartado creativo que invitaba al debate, pues no había una única solución correcta. Por todo ello, supusimos que sería una actividad muy adecuada para complementar las clases teóricas y que tenía factores que propiciaban la comunicación y cooperación entre los miembros del equipo, ya que el correcto entendimiento de un apartado del trabajo requería de la compresión de los apartados anteriores. Además la hipótesis de partida interrelacionaba varios bloques del temario de la asignatura, por lo que promovía una integración y visión global de la fisiología humana.

Durante todo el proceso los estudiantes estuvieron tutelados y guiados por el profesor, quien era responsable de orientarlos sobre aspectos como dónde buscar la información o cómo seleccionarla. Los estudiantes debían aprender a buscar la información de manera correcta, extraer lo más importante y compartirlo con el resto de compañeros, favoreciendo así el autoaprendizaje y el trabajo cooperativo.

\section{Objetivos}

El objetivo general de nuestro estudio era analizar si la actividad de los seminarios planteada como la redacción de un proyecto de investigación conseguía reforzar el aprendizaje de una manera cooperativa trabajando en grupo. El primer objetivo concreto era que el alumno interiorizase una serie de conceptos impartidos en clase que tenían relación con el trabajo planteado, mediante la búsqueda crítica de información contrastada y la transmisión de ésta al resto del equipo. En segundo lugar, mediante el trabajo en grupo se fomentaba el desarrollo de habilidades de comunicación y la defensa de las ideas. Por último, para tener una visión más completa de la actividad pedimos a los estudiantes pros y contras de la misma.

\section{Desarrollo de la innovación}

El estudio se llevó a cabo en la asignatura de Fisiología impartida durante el primer cuatrimestre del primer curso del Grado de Podología de la Universitat de Valencia, que contaba con 79 alumnos matriculados; 59 de ellos realizaron la actividad propuesta divididos en 11 grupos de 4 personas y 3 grupos de 5 .

Para comprobar el primer objetivo, si se habían adquirido los conocimientos mínimos, se realizó una prueba control que contenía tres preguntas básicas sobre conceptos fisiológicos que se habían trabajado con la actividad propuesta. Dicho control fue individual y tuvo lugar el día del examen final (primera convocatoria).

Para analizar el grado de participación en grupo (segundo objetivo) se realizó una encuesta privada a los estudiantes donde se les pedía el número de reuniones que habían

(cc)) BY-NC-ND 2016, Universitat Politècnica de València

Congreso In-Red (2016) 
destinado a la actividad y una valoración individualizada del resto de compañeros atendiendo a la asistencia a dichas reuniones y el material con el que habían contribuido. En base a estos criterios cada alumno obtuvo por parte de sus compañeros de grupo una calificación del 1 al 10. Por último y con la finalidad de obtener la opinión de los estudiantes sobre la actividad se les preguntó qué les había aportado realizar el trabajo en grupo: pros y contras. Las preguntas que contenía la encuesta se detalla a continuación:

1. ¿Cuantas reuniones de grupo habéis hecho?

a) De $1-5$

b) De 5-10

c) Más de 10

2. Indica en qué grado ha participado cada miembro basándote en las reuniones a las que ha acudido y el material que ha aportado al grupo. Escribe el nombre y primer apellido de cada uno de tus compañeros de grupo y puntúa en una escala del 1 a 10 su grado de participación.

Nombre y primer apellido $1-10$

3. Indica a tu juicio, los pros y contras que le has detectado a la actividad

\section{Resultados}

Un total de 52 estudiantes contestaron a la prueba de control de conocimientos y a la encuesta. La prueba control de conocimientos obtuvo 24 suspensos, 15 aprobados, 9 notables y 4 sobresalientes, uno de ellos con una puntuación de 10. (Tabla 1) Si estos resultados los comparamos con las calificaciones correspondientes al examen final en la primera convocatoria obtenemos que de los 79 alumnos matriculados, 65 se presentaron al examen final y las calificaciones fueron 28 suspensos, 18 aprobados, 17 notables y 2 sobresalientes. (Tabla 1) Los manuscritos presentados por los 14 grupos se evaluaron siguiendo una rúbrica elaborada por el equipo docente en la que se consideraban la estructura y el contenido. El contenido obtuvo una nota media de notable (7), con 4 grupos suspendidos, 2 aprobados, 3 notables y 5 sobresalientes. (Tabla 1)

La calificación que obtenía cada alumno por parte de sus compañeros de grupo fue de media un notable (8.0). Cabe destacar que 52 alumnos respondieron a la encuesta, pero

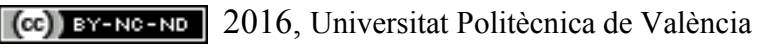


se obtuvieron las calificaciones correspondientes a 57 estudiantes, por lo que los 5 alumnos que no se presentaron al examen no calificaron pero sí fueron puntuados por sus compañeros. Hubo 30 sobresalientes, todos con una puntuación de 9 y sólo un alumno obtuvo una puntuación de 10 unánime por parte de sus compañeros de equipo. El resto fueron 20 notables, 6 aprobados y un suspenso. (Tabla 1)

En respuesta a la primera pregunta de la encuesta, un total de 34 alumnos afirmaron haberse reunido de 1 a 5 veces, 16 estudiantes respondieron que se habían reunido entre 5 y 10 veces para realizar el trabajo grupal y sólo 2 alumnos contestaron que se habían realizado más de 10 reuniones. Para verificar las respuestas se analizaron todas las que pertenecían a un mismo grupo y se observó que el $80 \%$ coincidía.

Tabla 1. Calificaciones de la prueba control de conocimientos, del examen final (primera convocatoria), nota obtenida en el manuscrito y la puntuación otorgada por los compañeros de grupo.

\begin{tabular}{ccccc}
\hline & Suspenso & Aprobado & Notable & Sobresaliente \\
\hline $\begin{array}{c}\text { Prueba control } \\
(52 \text { alumnos })\end{array}$ & 24 alumnos & 15 alumnos & 9 alumnos & 4 alumnos \\
& $(46 \%)$ & $(29 \%)$ & $(17 \%)$ & $(8 \%)$ \\
$\begin{array}{c}\text { Examen final } \\
(65 \text { alumnos })\end{array}$ & 28 alumnos & 18 alumnos & 17 alumnos & 2 alumnos \\
$\begin{array}{c}\text { Manuscrito } \\
(14 \text { grupos })\end{array}$ & 4 grupos & 2 grupos & 3 grupos & 5 grupos \\
$\begin{array}{c}\text { Puntuación } \\
\text { otorgada por los } \\
\text { compañeros }\end{array}$ & $129 \%)$ & $(14 \%)$ & $(21 \%)$ & $(36 \%)$ \\
$(57$ alumnos $)$ & $(2 \%)$ & $(10 \%)$ & $(35 \%)$ & $(53 \%)$ \\
\hline
\end{tabular}

La encuesta también reflejó los pros (tabla 2) y contras (tabla 3) de la actividad a juicio de los estudiantes. El mayor beneficio que destacaron (38 alumnos) fue el aprendizaje de conceptos, afianzamiento de lo impartido en teoría y la resolución de dudas entre sus iguales, seguido del beneficio de aprender a dialogar y debatir sobre un tema exponiendo las ideas propias y escuchando las de los demás ( 23 alumnos). En tercer lugar, 17 alumnos coincidían en que era más rápido y eficaz trabajar en grupo que de forma individual. Otros 14 estudiantes consideraron positivo el factor social de trabajar en grupo, por establecer relación con otros estudiantes y conseguir en algunos casos iniciar una amistad. Trece alumnos afirmaron que trabajar en grupo era gratificante, especialmente cuando todos los miembros tenían el mismo grado de implicación y similar manera de trabajar. Ocho alumnos hicieron alusión a lo "entretenido" que resultaba el trabajo en grupo si lo comparábamos con el individual. Ocho alumnos más afirmaron que les había servido para aprender a buscar información. Otras tres personas afirmaron haber mejorado su capacidad para organizarse gracias a esta actividad. Tres estudiantes respondieron que con

(c)) EY-NC-ND 2016, Universitat Politècnica de València

Congreso In-Red (2016) 
esta actividad se habían "animado" a hacerlo bien, ya que la nota de sus compañeros dependía de ellos.

Como aspectos negativos, la mayoría apuntó al tiempo invertido, muy seguido de la participación desigual de los integrantes del equipo y de las dificultades para reunirse. Quince alumnos vieron negativo el tener que discutir cómo hacer el trabajo con sus compañeros (qué incluir y cómo redactarlo). Seis alumnos se refirieron a la complicación del trabajo, cuatro destacaron como aspecto negativo el haberse enfadado con compañeros que no cooperaban y tres estudiantes se quejaron de no poder llevar su propio ritmo y verse atrasados por los demás.

Tabla 2. Pros de la actividad según los alumnos

\begin{tabular}{ll}
\hline Pros & $\mathrm{N}^{\circ}$ Alumnos \\
\hline Aprender y resolver dudas entre compañeros & 38 \\
Aprender a dialogar, saber escuchar y debatir ideas & 23 \\
Es más rápido y eficaz trabajar en grupo que de forma individual & 17 \\
Fomenta la relación con los compañeros y la amistad & 14 \\
Trabajar en grupo es gratificante & 13 \\
Más entretenido y ameno que el trabajo individual & 8 \\
Aprender a buscar información & 8 \\
Capacidad de organización & 5 \\
Te animas a hacerlo bien porque, en cierta medida, depende de ti la nota de & 3 \\
tus compañeros & \\
Se consigue un mejor resultado que si el trabajo hubiera sido individual & 2 \\
El trabajo me ha aportado paciencia & 2 \\
Me ha aportado ser menos cabezota & 2 \\
Mejorar la expresión oral & 2 \\
Me ha gustado que se plantease como un trabajo de investigación & 2 \\
Confiar en el trabajo de los demás & 1 \\
Aprender a sintetizar la información y quedarse con lo importante & 1 \\
Aprender a exponer ideas & 1 \\
No juzgar a mis compañeros por la primera impresión & 1 \\
\hline
\end{tabular}

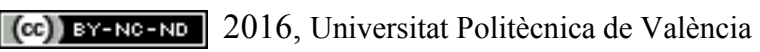


Tabla 3. Contras de la actividad según los alumnos

\begin{tabular}{ll}
\hline Contras & $\mathrm{N}^{\circ}$ Alumnos \\
\hline Mucho tiempo invertido & 21 \\
Participación desigual de los integrantes del equipo & 20 \\
Dificultad para reunirse & 19 \\
Discutir por cómo hacer el trabajo (qué incluir, cómo redactarlo ...) & 15 \\
No poder estar todos en las reuniones & 6 \\
Tema complicado, no tener claro por dónde empezar & 6 \\
Enfadarse con aquellos compañeros que no cooperan & 4 \\
No puedes llevar tu propio ritmo, dependes del resto del grupo & 3 \\
Tu nota depende del resto & 2 \\
$\begin{array}{l}\text { Siempre hay alguien en el grupo que quiere hacerlo todo y no consulta } \\
\text { con el resto }\end{array}$ & 2 \\
Mis compañeros tenían demasiada prisa por terminar el trabajo & \\
Si fuera posible, lo haría con el material real & 1 \\
\hline
\end{tabular}

\section{Conclusiones}

Aprender fisiología a través de la redacción de un proyecto de investigación, era la actividad propuesta para que nuestros alumnos reforzaran los conocimientos impartidos en clase y comenzaran a trabajar en grupo. Este estudio recoge las calificaciones individuales obtenidas en la prueba control, la nota correspondiente al examen final, la puntuación del manuscrito elaborado en consenso por cada grupo, la implicación de cada alumno en el equipo valorada por el resto de miembros y los pros y contras de la actividad a juicio de los estudiantes. Tomando los resultados en su conjunto, podemos concluir que el trabajo en grupo consigue mejores resultados que el individual, refuerza el aprendizaje, fomenta el diálogo y el debate de ideas entre iguales, resulta gratificante para los alumnos, les ayuda a aprender a cómo buscar la información y en algunos casos favorece la capacidad de organización.

En primer lugar, la prueba control sobre los conocimientos básicos que tenían que haber adquirido y afianzado con la actividad grupal, fue superada por el 54\% del alumnado. Si comparamos estas calificaciones con las obtenidas en el examen final de la asignatura (nota que refleja el estudio individual), se observa que el porcentaje de aprobados, un 57\% fue casi idéntico en ambas pruebas. Con estos datos, podíamos concluir que el aprendizaje en grupo fue similar al aprendizaje individual. No obstante, la motivación intrínseca de cada alumno es distinta y estos datos podrían reflejar que entre un 54-57\% del alumnado no tenía suficiente motivación por la asignatura en general y por tanto, ni trabajó individualmente para conseguir aprobar el examen final, ni trabajó lo suficiente en el grupo

(cc) EY-NC-ND 2016, Universitat Politècnica de València

Congreso In-Red (2016) 
para beneficiarse del aprendizaje cooperativo. Si analizamos el porcentaje de sobresalientes, observamos que la prueba control (representativa del aprendizaje en grupo) obtiene un $8 \%$ y el examen final (representativo del estudio individual) un 3\%. Por lo que podemos concluir que el aprendizaje de los conceptos trabajados en grupo se refuerza en aquellos alumnos que tienen una motivación intrínseca mayor. Esta observación señala la necesidad de desarrollar técnicas por parte de los decentes para aumentar la motivación de nuestros alumnos.

En segundo lugar, la comparativa entre las puntuaciones obtenidas en los manuscritos y las notas de la prueba control de conocimientos, demuestra que el trabajo en grupo consigue mejores resultados (el $71 \%$ de los grupos aprueba el manuscrito, con un $36 \%$ de sobresalientes) que el trabajo individual (el 54\% de los estudiantes aprueba el control de conocimientos con un $8 \%$ de sobresalientes). Por tanto, en cuanto a la tarea solicitada, el resultado es de mayor calidad si el trabajo se hace en equipo. Aunque, bien es cierto que esto podría deberse a la existencia de buenos estudiantes que se responsabilizan en mayor medida y consiguen redactar un buen trabajo para todo el grupo. Si no se hubiera realizado la prueba control individual no se hubiera detectado este aspecto. Así pues, se puede concluir que hacer este control individual es necesario para evaluar más justamente los conocimientos adquiridos por cada alumno.

En tercer lugar, el análisis de las encuestas reveló, según el criterio de los estudiantes, que la actividad les reporta más pros que contras. Para valorar el grado de sinceridad con respecto a la pregunta 1 de la encuesta, comparamos las contestaciones de los miembros de un mismo grupo y el $80 \%$ coincidía, es decir habían contestado lo mismo, por lo que era fiable dicha información. Los resultados de la encuesta muestran que la actividad propuesta consiguió que los alumnos dialogasen entre ellos para llegar a un consenso en la redacción del manuscrito, según la propia opinión del alumnado, que también afirmó que la actividad había resultado útil para resolver dudas entre compañeros, lo que implica que se ha potenciado el aprendizaje cooperativo.

El tiempo preocupa mucho a nuestros estudiantes, de hecho los alumnos afirman que el trabajo en grupo es más rápido y eficaz, esta respuesta indica que sí hay un porcentaje de alumnos que verdaderamente se han beneficiado de trabajar en grupo y reconocen que no hubieran hecho un trabajo tan bueno en tan poco tiempo de manera individual. Además el trabajar en grupo resultaba "entretenido", incluso alguno contestó "divertido". Hay que destacar que si el aprendizaje se hace de una manera lúdica aumenta la motivación por la asignatura como hemos demostrado en estudios previos (Mauricio, 2015)

Uno de los puntos débiles de nuestro alumnado es que no saben filtrar la información, por eso el docente invierte tiempo en este apartado, así que el hecho de que algunos consigan aprender esta habilidad, como ellos mismo refieren, nos es muy grato.

Un dato curioso y digno de resaltar fue que tres estudiantes se hubiesen "animado" a hacer bien el trabajo porque, en cierta medida, la nota de sus compañeros dependía de ellos, especialmente cuando la respuesta de otros dos estudiantes fue la misma pero desde el

(c)) EY-NC-ND 2016, Universitat Politècnica de València 
punto de vista opuesto, es decir, veían como aspecto negativo que su nota dependiera de los demás. Con estos datos, podríamos afirmar que la actividad puede fomentar la responsabilidad en ciertos alumnos con mayor grado de solidaridad.

Como aspectos negativos, la mayoría apuntó al tiempo invertido, muy seguido de la participación desigual de los integrantes y de las dificultades para reunirse. Quince alumnos vieron negativo el tener que discutir aspectos sobre el trabajo con sus compañeros. Probablemente se tratase de grupos con miembros no compatibles ya que uno de los pros más destacados fue conseguir el debate y el consenso dentro del equipo, objetivo fundamental de la actividad. Cabe aclarar que dada la brevedad de las asignaturas cuatrimestrales, como la nuestra, los alumnos han de ser agrupados por el profesor, la primera semana de clase y considerando que se trata de una asignatura de primer cuatrimestre de primer curso, los estudiantes no se conocían previamente.

Seis alumnos nos transmitieron su angustia por ir demasiado perdidos, especialmente al principio, cosa que los docentes ya habíamos notado por el elevado número de tutorías solicitadas por dicho motivo. Nos fue grato encontrar en las encuestas que la dificultad del tema se había disipado a medida que habían empezado a avanzar en el trabajo y finalmente sólo seis alumnos refirieron este aspecto como un contra.

A la hora de calificar a sus compañeros se detectó que existía una tendencia a evaluar positivamente a los demás, por lo que se desprende un buen clima de compañerismo. La mayoría, el 53\% fueron calificados por sus iguales con un sobresaliente. La nota media obtenida fue de notable (8). A pesar de este ambiente amigable se detectaron casos en los que los alumnos no habían trabajado verdaderamente en grupo (bien por no asistir a la reuniones, bien por no aportar el material suficiente) y sus compañeros los habían evaluado por debajo de la media, es decir con un aprobado o con un suspenso. Estos casos, que fueron 7, (6 aprobados y un suspenso) se analizaron más detenidamente. Se observó que los seis alumnos calificados con un aprobado por sus iguales o bien no se presentaron o bien suspendieron la prueba control, por lo que estos resultados están en concordancia, pues dichos alumnos no mostraron interés ni por el trabajo grupal ni por la asignatura. Es curioso que el único estudiante que fue suspendido por sus compañeros de equipo, obtuviera una calificación en la prueba control de 5.5 y en el examen final de 8.8. Estas dos notas no están en concordancia y ponen de manifiesto que efectivamente se trataba de un estudiante con capacidad individual para obtener una buena nota, pero no había rendido en el grupo lo suficiente para sus compañeros, quienes lo penalizaron en la encuesta y además quedó patente que así había sido con la calificación de la prueba control. Nuestro análisis pone de manifiesto que tanto la evaluación de cada estudiante por sus iguales como la prueba control son elementos válidos para ser considerados en la evaluación de actividades grupales.

Del presente estudio se desprende que el hecho de trabajar en grupo resulta muy difícil para nuestros alumnos de primer curso, probablemente por estar acostumbrados a un sistema educativo individualista en el cual la nota depende del esfuerzo personal de cada uno y no del esfuerzo colectivo. Sin embargo, les reporta muchos beneficios, como ellos mismos refieren, destacando el aprendizaje y resolución de dudas entre compañeros y el

2016, Universitat Politècnica de València

Congreso In-Red (2016) 
debate de ideas, aprendiendo a expresar las propias y escuchar las de los demás. Por todo ello, consideramos que este tipo de actividades ayuda a los estudiantes a desarrollar unas competencias necesarias para su futuro laboral. Además nuestro análisis revela que sólo aquellos estudiantes con una motivación intrínseca mayor por la asignatura son capaces de beneficiarse del aprendizaje cooperativo, lo que implica la necesidad de seguir realizando actividades como esta e ir perfeccionándolas para lograr beneficios en un mayor número de alumnos.

\section{Referencias}

CROOKS, T. J. "The impact of classroom evaluation practices on students" en Review of Educational Research. (1988, 58, 438-481)

JOHNSON, D. W. "Effects of cooperative, competitive, and individualistic goal structures on achievement: A meta-analysis" en Psychological Bulletin. (1981, 89, 47-62)

JOHNSON DW, JOHNSON RT, HOLUBEC EJ, VITALE G. (1999) Aprendizaje cooperativo en el aula. Buenos Aires, Argentina. Editorial Paidós Ibérica SA

MAURICIO, M.D, SERNA, E y VALLES, S.L. (2015) "Experiencias en la aplicación de la gamificación en $1^{\circ}$ Curso de Grado de Ciencias de la Salud" en Congreso de Innovación Educativa y Docencia en Red. Universitat Politècnica de València. Disponible en $<$ http://ocs.editorial.upv.es/index.php/INRED/>

ROVAI A.P. "Development of an instrument to measure classroom community" en The Internet and Higher Education. (2002, 5,197-211) 